\title{
The leptonic CP phase is determined by an equation involving the PMNS matrix elements
}

\author{
Hong-Wei $\mathrm{Ke}^{1 *}$, Jia-Hui Zhou and Xue-Qian $\mathrm{Li}^{2 \dagger}$ \\ ${ }^{1}$ School of Science, Tianjin University, Tianjin 300072, China \\ 2 School of Physics, Nankai University, Tianjin 300071, China
}

\begin{abstract}
Several approximate equalities among the matrix elements of CKM and PMNS imply that hidden symmetries may exist and be common for both quark and neutrino sectors. The CP phase of the CKM matrix $\left(\delta_{\mathrm{CKM}}\right)$ is involved in these equalities and can be investigated when these equalities turn into several equations. As we substitute those experimentally measured values of the three mixing angles into the equations for quarks, it is noted that one of the equations which holds exactly has a solution $\delta_{\mathrm{CKM}}=68.95^{\circ}$. That value accords with $\left(69.1_{-3.85}^{+2.02}\right)^{\circ}$ determined from available data. Generalizing the scenario to the lepton sector, the same equality determines the leptonic CP phase $\delta_{\mathrm{PMNS}}$ to be $276.10^{\circ}$. Thus we predict the value of $\delta_{\mathrm{PMNS}}$ from the equation. So far there is no direct measurement on $\delta_{\text {PMNS }}$ yet, but a recent analysis based on the neutrino oscillation data prefers the phase close to $270^{\circ}$.
\end{abstract}

PACS numbers: 12.15.Ff, 14.60.Pq, 14.60.Lm

\section{INTRODUCTION}

Following recognition of quark mixing and determining the non-zero $\mathrm{CP}$ phase in the quark sector, with longtime accumulation of data on solar[1-3], atmosphere [4, 5], accelerator [68] and reactor neutrino experiments $9-14$, the mixing among different neutrino flavors is confirmed. Even 5 to 10 years before the first measurement on the solar neutrino flux had been made, the neutrino mixing [15, 16] was proposed and with the picture 17], the solar neutrino flux deficit measured fifty years ago can be naturally interpreted. Since the lepton flavor eigenstates do not match with the mass eigenstates a unitary transformation matrix i.e. Pontecorvo-Maki-NakawagaSakata (PMNS) matrix was introduced to bridge them. The $3 \times 3$ matrix possesses three mixing angles and a $\mathrm{CP}$ phase which may also be non-zero. From then on, to determine the mixing parameters and $\mathrm{CP}$ phase composes the most important task for both experimentalists and theorists of this field. Even though through hard work the three mixing angles have been determined with a certain accuracy, the leptonic CP phase is still not clear. Along with the experimental search for the $\mathrm{CP}$ pase, one might expect to predict it from the theory aspect.

In our early works 18, 19] several approximate equalities among the matrix elements of Cabibbo-Kobayashi-Maskawa $(\mathrm{CKM})[20,21]$ were found based on investigating relations among different parametrization schemes of the mixing matrix (CKM and PMNS). The equalities may be induced by a hidden symmetry. It is noted that those equalities also hold for the lepton sector, even though the present data on neutrinos are not as accurate as for the quark sector. Obviously those equalities may help to reduce the number of free parameters of the matices, i.e. the degree of freedom is reduced due to existence of the hidden symmetry. For that we regard these equalities to several equations. Supposing these equations to be exact, by inputting several CKM matrix elements[22, 23]

\footnotetext{
*khw020056@hotmail.com, Corresponding author.

†lixq@nankai.edu.cn
}

into the equations, other elements are obtained, and it is found that the results well coincide with the available data within the error tolerance range. In the three-generation fermion framework, there are only four free parameters in the $3 \times 3$ mixing matrix i.e. the three mixing angles and one $\mathrm{CP}$ phase, thus we expect that due to a hidden symmetry one or more parameters could be determined by the other parameters via the equations. In this work, we are able to determine the CP phase of CKM from the equations and then generalize this scenario to the lepton sector. Meanwhile, we would testify which equality holds more precisely or in other words, to judge their approximation degrees. The results help us to conclude whether the hidden symmetry is complete or somehow slightly broken. In this paper we will use simplified forms of these equations which were given in our earlier works and investigate the values of the leptonic CP phase by these equations.

\section{EQUALITIES AMONG THE MATRIX ELEMENTS AND THE CP PHASE}

Mixing among different flavors of quarks (neutrinos) via the CKM (PMNS) matrix has been firmly recognized. The Lagrangian of the weak interaction reads

$\mathcal{L}=\frac{g}{\sqrt{2}} \bar{U}_{L} \gamma^{\mu} V_{\mathrm{CKM}} D_{L} W_{\mu}^{+}+\frac{g}{\sqrt{2}} \bar{E}_{L} \gamma^{\mu} V_{\mathrm{PMNS}} N_{L} W_{\mu}^{-}+$h.c., (1)

where $U_{L}=\left(u_{L}, c_{L}, t_{L}\right)^{T}, D_{L}=\left(d_{L}, s_{L}, b_{L}\right)^{T}, E_{L}=$ $\left(e_{L}, \mu_{L}, \tau_{L}\right)^{T}$ and $N_{L}=\left(v_{1}, v_{2}, v_{3}\right)^{T} . V_{\mathrm{CKM}}$ and $V_{\mathrm{PMNS}}$ are the $\mathrm{CKM}$ and PMNS matrices respectively. The $3 \times 3$ mixing matrix $V$ is written as

$$
V=\left(\begin{array}{lll}
V_{11} & V_{12} & V_{13} \\
V_{21} & V_{22} & V_{23} \\
V_{31} & V_{32} & V_{33}
\end{array}\right)
$$

Generally, for a $3 \times 3$ unitary matrix there are four independent parameters, namely three mixing angles and one $\mathrm{CP}$ phase. There can be various schemes to parameterize the matrix which are summarized in Ref.[24]. The standard 
parametrization is shown as 22]

$$
V=\left(\begin{array}{ccc}
c_{12} c_{13} & s_{12} c_{13} & s_{13} \\
-c_{12} s_{23} s_{13}-s_{12} c_{23} e^{i \delta} & -s_{12} s_{23} s_{13}+c_{12} c_{23} e^{i \delta} & s_{23} c_{13} \\
-c_{12} s_{23} s_{13}+s_{12} s_{23} e^{i \delta} & -s_{12} s_{23} s_{13}-c_{12} s_{23} e^{i \delta} & c_{23} c_{13}
\end{array}\right)
$$

Here $s_{j k}$ and $c_{j k}$ denote $\sin \theta_{j k}$ and $\cos \theta_{j k}$ with $j, k=1,2,3$. $\delta$ is the CP phase and $\delta_{\mathrm{CKM}}$ and $\delta_{\mathrm{PMNS}}$ will be used for CKM and PMNS respectively.

In our previous work [18, 19] definite relations among the values of $\sin \delta_{i}$ where $\delta_{i}$ 's are the CP phase in the nine different parametrization schemes were found, then we turned these relations into several approximate equalities among the matrix elements of CKM (or PMNS).

These equalities can be then rewritten to more succinct forms

$$
\begin{gathered}
\frac{\left|V_{21}\right|\left|V_{22}\right|}{1-\left|V_{23}\right|^{2}}-\frac{\left|V_{11}\right|\left|V_{12}\right|}{1-\left|V_{13}\right|^{2}} \approx 0, \\
\frac{\left|V_{11}\right|\left|V_{12}\right|\left|V_{21}\right|}{1-\left|V_{11}\right|^{2}}-\frac{\left|V_{23}\right|\left|V_{32}\right|\left|V_{33}\right|}{1-\left|V_{33}\right|^{2}} \approx 0, \\
\frac{\left|V_{23}\right|\left|V_{33}\right|}{1-\left|V_{13}\right|^{2}}-\frac{\left|V_{22}\right|\left|V_{32}\right|}{1-\left|V_{12}\right|^{2}} \approx 0, \\
\frac{\left|V_{12}\right|\left|V_{22}\right|}{1-\left|V_{32}\right|^{2}}-\frac{\left|V_{11}\right|\left|V_{21}\right|}{1-\left|V_{31}\right|^{2}} \approx 0 \\
\frac{\left|V_{22}\right|\left|V_{23}\right|}{1-\left|V_{21}\right|^{2}}-\frac{\left|V_{32}\right|\left|V_{33}\right|}{1-\left|V_{31}\right|^{2}} \approx 0
\end{gathered}
$$

where normalization conditions such as $\left|V_{13}\right|^{2}+\left|V_{23}\right|^{2}+\left|V_{33}\right|^{2}=$ 1 were used and $\left|V_{j k}\right|(j, k=1,2,3)$ are the modules of the corresponding matrix elements. Apparently Eqs. (4) and (8) display relations among the matrix elements of the two adjacent rows and Eqs. (6) and (7) demonstrate relations among the matrix elements of the two adjacent columns. Eq. (5) corresponds to the relations among the six matrix elements $\left|V_{11}\right|,\left|V_{12}\right|,\left|V_{21}\right|,\left|V_{23}\right|,\left|V_{32}\right|$ and $\left|V_{33}\right|$. These equalities reveal existence of some underlying symmetries which determine them.

Now let us suppose the matrix elements to be variables, then these equalities would turn into several equations by which one may expect to gain solutions. In this letter we will further investigate the CP phases of CKM and PMNS in terms of the corresponding equations. Since there are abundant data on the hadron decays, the parameters in the CKM matrix are well determined. One can use these values to judge which equality holds with higher precision.

Using the expressions of matrix elements in Eq. (3), Eqs. (4)-(8) are transformed into the forms directly related to the three mixing angles and $\mathrm{CP}$ phase. If one inputs the values of three mixing angles he can study the dependence of the left sides of Eqs. (4)-(8) on the CP phase. For quark sector the dependence is depicted in Fig. 1(a)-(e) with $\theta_{12}=13.023^{\circ}, \theta_{23}=$ $2.360^{\circ}, \theta_{13}=0.201^{\circ}$ [24]. From those diagrams, one finds:
TABLE I: The values of the CP phase which make the five equations to hold more accurately.

\begin{tabular}{ccc|cc}
\hline \hline Eq. & $\delta_{\text {CKM }}(1)$ & $\delta_{\text {CKM }}(2)$ & $\delta_{\text {PMNS }}(1)$ & $\delta_{\text {PMNS }}(2)$ \\
\hline$(4)$ & $90.01^{\circ}$ & $269.98^{\circ}$ & $93.21^{\circ}$ & $266.79^{\circ}$ \\
\hline 5 & $68.95^{\circ}$ & $291.05^{\circ}$ & $83.90^{\circ}$ & $276.10^{\circ}$ \\
\hline 6 & $90.57^{\circ}$ & $269.43^{\circ}$ & $90.74^{\circ}$ & $269.26^{\circ}$ \\
$(7$ & $0^{\circ}$ & $360^{\circ}$ & $0^{\circ}$ & $360^{\circ}$ \\
\hline 8 & $0^{\circ}$ & $360^{\circ}$ & $0^{\circ}$ & $360^{\circ}$ \\
\hline \hline
\end{tabular}

1. All the left sides of the five equations vary within very small ranges for different $\delta_{\mathrm{CKM}}$ values.

2. At the two sultions the first three equations hold exactly. We list the corresponding values in Table凹

3. The last two equations do not possess solutions which are reasonable, namely the values of the left sides of the equations are close to 0 when $\delta_{\mathrm{CKM}}=0^{\circ}$ or $360^{\circ}$ that obviously contradict to the suggested data.

4. The left side of Eq. (5) is sensitive to the change of $\delta_{\mathrm{CKM}}$.

5. The solution $68.95^{\circ}$ obtained from Eq. (5) is accordant with the data $\left(69.1_{-3.85}^{+2.02}\right)^{\circ}[24]$ within the error tolerance i.e. the equality offers proper information on the hadronic $\mathrm{CP}$ phase for the quark sector.

Apparently Eq. (5) holds with the highest accuracy whereas Eqs. (4) and (6) are not so precise, but the approximation degree is still appreciable, then for expressions of (7) and (8) equality is almost totally lost to a not-allowed degree. Thus it means that the equality degree of Eq. (4) through Eq. (8) could be different as the underlying symmetry is broken, therefore not all of them can be applied to determine the $\mathrm{CP}$ phase by inputting the mixing angles. We decide that Eq. (5) would be the best choice.

So far there is no direct measurement on the leptonic CP phase yet, naturally one may expect to gain information on it from future experiments. It is known that $\delta_{\mathrm{PMNS}}$ can induce $\mathrm{CP}$ violation effects in neutrino oscillation experiments. Better than nothing, at present, indirect analyses [25, 26] based on the neutrino experiments suggest that the leptonic $\mathrm{CP}$ phase $\delta_{\text {PMNS }}$ is close to $270^{\circ}$. Since the Eq. (5) predicts a more precise value of $\delta_{\mathrm{CKM}}$, we have all reason to expect that it is eligible to be applied for determining $\delta_{\text {PMNS }}$. In analog to the quark case as inputting the central values of $\theta_{12}=33.71^{\circ}, \theta_{23}=$ $41.38^{\circ}, \theta_{13}=8.80^{\circ}$ (for normal ordering of neutrino masses) one can fix $\delta_{\text {PMNS }}=83.90^{\circ}$ or $276.10^{\circ}$ as Eq. (5) is supposed to hold exactly. For inverted ordering of neutrino masses the central value of $\theta_{13}$ is $8.91^{\circ}$ and then the predicted value of $\delta_{\text {PMNS }}$ would change only slightly. Apparently the theoretical prediction $\delta_{\mathrm{PMNS}} \sim 276.10^{\circ}$ is consistent with the results given in Ref. [25, 26]. If the result is confirmed by the future measurements one would find that the sum of $\delta_{\mathrm{CKM}}$ and $\delta_{\text {PMNS }}$ is close to $360^{\circ}$ i.e. $\delta_{\mathrm{CKM}}$ adopts the left side value of Fig. 11 b) whereas $\delta_{\text {PMNS }}$ takes the right side value (f). The mutual complementarity between $\delta_{\text {PMNS }}$ and $\delta_{\text {CKM }}$ may impose a constrant condition to exclude the solution of $83.90^{\circ}$. From Tab. Ione can note that the solutions obtained from Eqs. (4) and (6) are also close to $270^{\circ}$, even though in the quark sector the $\mathrm{CP}$ phase determined by their counterpart equations 


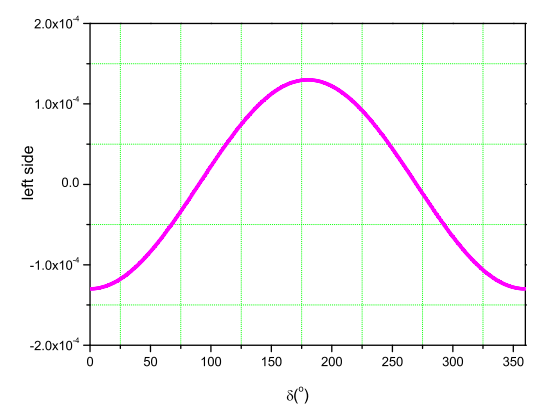

(a) Eq. (9) (CKM)

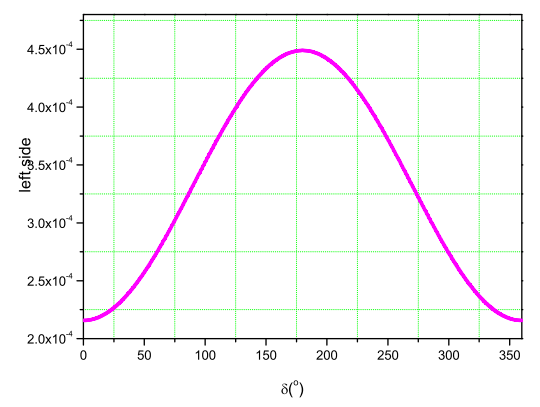

(d) Eq. (12) (CKM)

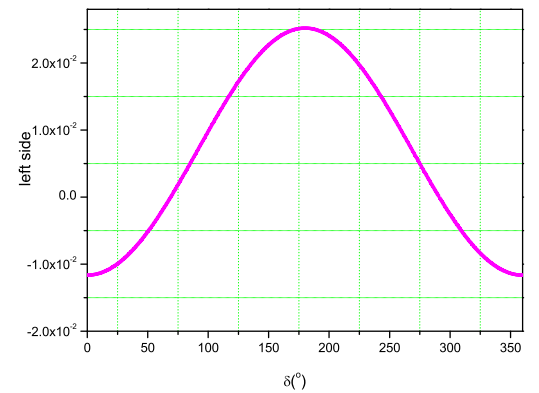

(b) Eq. (10) (CKM)

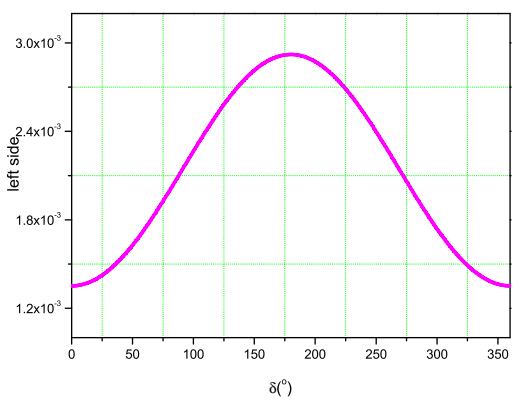

(e) Eq. (13) (CKM)

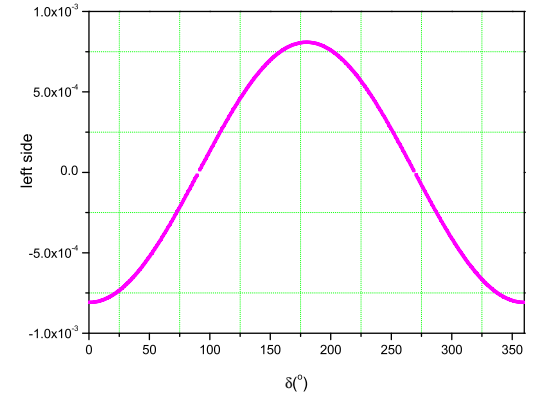

(c) Eq. (11) (CKM)

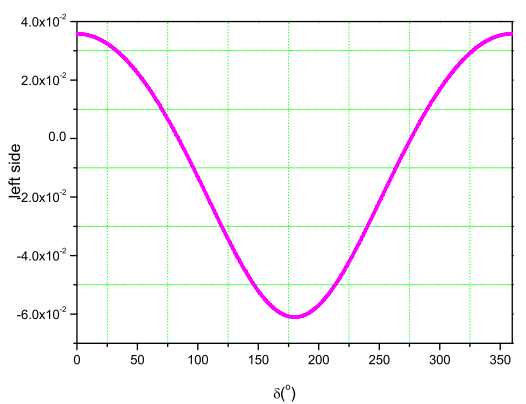

(f) Eq. (10) (PMNS)

FIG. 1: The dependence of the left sides of Eq. (4)-Eq. (8) on the CP phase $\delta$

apparently deviates from data.

\section{SUMMARY}

In our previous paper several approximate equalities among the elements of CKM (PMNS) were derived. In this letter we try to simplify them and those simple and succinct relations imply existence of hidden symmetries in both the quark and neutrino sectors even though they may be approximate or slightly broken. Using the equations we investigate the $\mathrm{CP}$ phase of CKM and PMNS matrices. Supposing these equations hold exactly, as the three mixing angles of quarks are inputs one only obtains an accurate $\delta_{\mathrm{CKM}}$ from Eq. (5) whereas the others equations fail to provide precise information about the CP phase, even though the solutions of Eq. (4) and Eq. (6) are still plausibly close to the data. By solving Eq. (5) with inputting the the measured values of the neutrino mixing angles we obtain the leptonic CP phase $\delta_{\text {PMNS }}$ to be $83.90^{\circ}$ or $276.10^{\circ}$. Since at present there is no direct experimental measurement on $\delta_{\text {PMNS }}$ of PMNS yet, we need to determine which value of the two would be that chosen by the nature. The mutual complementarity between quark and lepton sectors help to pin it down. Moreover, indirect analyses made by experimentalists indicate that the phase is close to $270^{\circ}$. Thus the solution $\delta_{\mathrm{PMNS}}=276.10^{\circ}$ is consistent with the recent analyses on neutrino experiment.

It is well known that the $3 \times 3$ unitary matrix for fermion mixing can have three mixing angles and one $\mathrm{CP}$ phase. Generally, except the unitary requirement, it seems that there are no other constraints on them, so the four parameters are independent. Namely, one cannot derive any of them from the other three. However, our analysis clearly shows that from the equations which were derived in our earlier works the $\mathrm{CP}$ phase can be obtained from the three mixing angles whose values are experimentally measured. It implies that there is a hidden symmetry which may associate the four parameters together. Even though we do not know what the hidden symmetry is, we notice its existence. The hidden symmetry determines those equalities which we discovered from phenomenology. Therefore, precise data of the three mixing angles would determine the CP phase except there exist a $\delta$ and $2 \pi-\delta$ degeneracy. That is the common sense. Moreover, the association between quark and lepton sectors is exposed and this generalization of those equalities from quark sector to leptonic one is by no means trivial. In particular, we use the same equality which holds for both quarks and leptons to obtain the CP phase as $276.10^{\circ}$ or $83.90^{\circ}$ for neutrinos which has not well been experimentally measured yet but is somehow determined by the oscillation data, and our theoretical prediction is consistent with this value. In this sense, the degeneracy of $\delta$ and $2 \pi-\delta$ is lifted by the mutual complementarity between quark and lepton sectors as $\delta_{P M N S}=270^{\circ}$ being uniquely fixed. In this work we only expose this fact from the phenomenological aspect, but it is still mysterious if all the equalities indeed originate from a hidden symmetry. If so, 
does the phenomenon that some of the equalities are only approximately holding, mean breaking of the symmetry or there exists a mechanism which causes deviation from exact holding? The profound source is worth of further exploration.

\section{Acknowledgement}

This work is supported by the National Natural Science Foundation of China (NNSFC) under the contract No.
11375128 and 11135009.
[1] R. Davis, Jr., D. S. Harmer and K. C. Hoffman, Phys. Rev. Lett. 20, 1205 (1968).

[2] Q. R. Ahmad et al. [SNO Collaboration], Phys. Rev. Lett. 89, 011301 (2002).

[3] B. Aharmim et al. [SNO Collaboration], heavy-water detector at the Sudbury Neutrino Observatory," Phys. Rev. C 72 (2005) 055502 .

[4] Y. Fukuda et al. [Super-Kamiokande Collaboration], Phys. Rev. Lett. 81, 1562 (1998).

[5] Y. Ashie et al. [Super-Kamiokande Collaboration], Phys. Rev. Lett. 93, 101801 (2004).

[6] M. H. Ahn et al. [K2K Collaboration], Phys. Rev. Lett. 90, 041801 (2003).

[7] D. G. Michael et al. [MINOS Collaboration], Phys. Rev. Lett. 97, 191801 (2006).

[8] P. Adamson et al. [MINOS Collaboration], Phys. Rev. Lett. 101, 131802 (2008).

[9] K. Eguchi et al. [KamLAND Collaboration], Phys. Rev. Lett. 90, 021802 (2003).

[10] S. Abe et al. [KamLAND Collaboration], Phys. Rev. Lett. 100, 221803 (2008).

[11] Y. Abe et al. [Double Chooz Collaboration], Phys. Rev. Lett. 108, 131801 (2012) doi:10.1103/PhysRevLett.108.131801 [arXiv:1112.6353 [hep-ex]].

[12] Y. Abe et al. [Double Chooz Collaboration], Phys. Rev. $\begin{array}{llll}\text { D 86, } 052008 & \text { (2012) doi:10.1103/PhysRevD.86.052008 }\end{array}$ [arXiv:1207.6632 [hep-ex]].

[13] F. P. An et al. [Daya Bay Collaboration], Phys. Rev. Lett. 108, 171803 (2012) doi:10.1103/PhysRevLett.108.171803
arXiv:1203.1669[hep-ex]].

[14] J. K. Ahn et al. [RENO Collaboration], Phys. Rev. Lett. 108, 191802 (2012) doi:10.1103/PhysRevLett.108.191802 arXiv:1204.0626 [hep-ex]].

[15] B. Pontecorvo, Sov. Phys. JETP 6, 429 (1957) [Zh. Eksp. Teor. Fiz. 33, 549 (1957)]; Sov. Phys. JETP 7, 172 (1958) [Zh. Eksp. Teor. Fiz. 34, 247 (1957)].

[16] Z. Maki, M. Nakagawa and S. Sakata, Prog. Theor. Phys. 28, 870 (1962).

[17] B. Pontecorvo, Sov. Phys. JETP 26, 984 (1968) [Zh. Eksp. Teor. Fiz. 53, 1717 (1967)].

[18] H. W. Ke and X. Q. Li, Commun. Theor. Phys. 64, 519(2015).

[19] H. W. Ke, S. X. Zhao and X. Q. Li, Chinese Physics C 40, 053101(2016).

[20] N. Cabibbo, Phys. Rev. Lett. 10, 531 (1963).

[21] M. Kobayashi and T. Maskawa, Prog. Theor. Phys. 49, 652 (1973).

[22] K. A. Olive et al. [Particle Data Group Collaboration], Chin. Phys. C 38, 090001 (2014).

[23] X. W. Kang, B. Kubis, C. Hanhart and U. G. Mei?ner, Phys. Rev. D 89, 053015 (2014).

[24] Y. Zhang, X. Zhang and B. -Q. Ma, Phys. Rev. D 86, 093019 (2012) arXiv:1211.3198 [hep-ph]].

[25] K. Abe et al. [T2K Collaboration], Phys. Rev. Lett. 112, 061802 (2014).

[26] M. C. Gonzalez-Garcia, M. Maltoni and T. Schwetz, JHEP 1411, 052 (2014). 